\title{
Slow oscillations in neural networks with facilitating synapses
}

\author{
Ofer Melamed • Omri Barak • Gilad Silberberg • \\ Henry Markram • Misha Tsodyks
}

Received: 11 June 2007 /Revised: 20 January 2008 / Accepted: 23 January 2008 / Published online: 16 May 2008

(C) Springer Science + Business Media, LLC 2008

\begin{abstract}
The synchronous oscillatory activity characterizing many neurons in a network is often considered to be a mechanism for representing, binding, conveying, and organizing information. A number of models have been proposed to explain high-frequency oscillations, but the mechanisms that underlie slow oscillations are still unclear. Here, we show by means of analytical solutions and simulations that facilitating excitatory $\left(E_{\mathrm{f}}\right)$ synapses onto interneurons in a neural network play a fundamental role, not only in shaping the frequency of slow oscillations, but also in determining the form of the up and down states observed in electrophysiological measurements. Short time constants and strong $E_{\mathrm{f}}$ synapse-connectivity were found to induce rapid alternations between up and down states, whereas long time constants and weak $E_{\mathrm{f}}$ synapse connectivity prolonged the time between up states and increased the up state duration. These results suggest a novel role for facilitating
\end{abstract}

\section{Action Editor: Nicolas Brunel}

O. Melamed $(\bowtie)$

Department of Sciences, Holon Institute of Technology, Holon 58102, Israel

e-mail: ofer.melamed@hit.ac.il

O. Melamed $\cdot$ G. Silberberg $\cdot$ H. Markram

Laboratory of Neural Microcircuitry, Brain Mind Institute, EPFL, Lausanne 1015, Switzerland

O. Barak $\cdot$ M. Tsodyks

Department of Neurobiology, The Weizmann Institute of Science, Rehovot 76100, Israel

G. Silberberg

Nobel Institute for Neurophysiology,

Department of Neuroscience, Karolinska Institute,

17177 Stockholm, Sweden excitatory synapses onto interneurons in controlling the form and frequency of slow oscillations in neuronal circuits.

Keywords Dynamic synapse · Synchrony $\cdot$ Recurrent network $\cdot$ Temporal processing $\cdot$ Neocortex $\cdot$ Model

\section{Introduction}

The brain can display multiple forms and frequencies of oscillatory activity. Slow wave $(0.1-1 \mathrm{~Hz})$ oscillations recorded by an electroencephalogram (EEG) are manifested by reduced alertness, such as during sleep or anesthesia (Steriade et al. 1993a; Moruzzi and Magoun 1995). However, the manner by which this type of activity arises remains unclear. A series of combined EEG and intracellular in vivo measurements have provided empirical evidence that the phases of the oscillations recorded with an EEG are correlated with repeated bi-stable states, called $u p$ and down states, of the cortical intracellular electrical activity (Contreras and Steriade 1995). The up state is related to periods of relative depolarization and firing of cortical neurons. In contrast, the down state is associated with hyperpolarization or a silent phase (Steriade et al. 1993b, 1996; Stern et al. 1997; Lampl et al. 1999).

An open question is whether slow oscillations are driven by recurrent loops with distal brain areas, inter-columnar interactions, or the intrinsic properties of the local recurrent microcircuit. Slow oscillations have been observed in neocortical slices, suggesting that they could be induced by the cellular and synaptic properties of the local network (Sanchez-Vives and McCormick 2000). Such local networks contain both excitatory and inhibitory cells, which can form a negative feedback loop and thus constitute a candidate mechanism for the generation of oscillations. 
Since both the membrane and the synaptic time constants are usually on the order of several milliseconds, these oscillations are expected to be fast (above $40 \mathrm{~Hz}$ ). Slow mechanisms, such as intrinsic after-hyperpolarization currents (Timofeev et al. 2000; Compte et al. 2003), short-term synaptic depression (Timofeev et al. 2000) or slow synaptic currents (NMDA and $\mathrm{GABA}_{\mathrm{B}}$ ) (Parga and Abbott 2007) have been proposed to generate slow oscillations in a local circuit. It is known, however, that synapses from excitatory to inhibitory neurons can exhibit short term plasticity in the form of facilitation (Thomson et al. 1993; Markram et al. 1998). Such connections have a relatively long time constant (hundreds of milliseconds), and can therefore enable slow oscillations (below $5 \mathrm{~Hz}$ ) in the network. We examined networks of excitatory and inhibitory cells, where the connection from the excitatory to the inhibitory neurons is facilitating and found that slow oscillations indeed arise in such networks. We show the conditions and properties of such oscillations, and discuss their significance.

\section{Firing rate model of a recurrent neural network}

Here we considered a recurrent network consisting of excitatory and inhibitory populations with mean firing rates, $E$ and $I$, respectively. These populations are synaptically interconnected by four types of connections and receive constant external inputs to excitatory, $E_{0}$, and inhibitory, $I_{0}$, populations (Fig. 1(a)). This network describes a neocortical area consisting of pyramidal cells and interneurons. The following set of equations, based on a rate model, describe the dynamics of such a network (Wilson and Cowan 1972).

$$
\begin{aligned}
& \dot{E}=-\frac{E}{\tau_{\mathrm{e}}}+\frac{g\left(J_{\mathrm{ee}} E-J_{\mathrm{ei}} I+E_{0}\right)}{\tau_{\mathrm{e}}} \\
& \dot{I}=-\frac{I}{\tau_{\mathrm{i}}}+\frac{g\left(J_{\mathrm{ie}} E-J_{\mathrm{ii}} I+I_{0}\right)}{\tau_{\mathrm{i}}}
\end{aligned}
$$

where $\tau_{\mathrm{e}}\left(\tau_{\mathrm{i}}\right)$ is the time constant of the excitatory (inhibitory) response to synaptic inputs and $J_{a b}$ is the synaptic strength projection from population $b$ to $a$. In this study, we chose a threshold linear response function $g(x)$ :

$g(x)=\left\{\begin{array}{lr}0 & x<T \\ \beta \cdot(x-T) & x \geq T\end{array}\right.$

where $\beta$ and $T$ are the slope and the threshold, respectively.

\section{Facilitating synapses}

To study the influence of facilitating synapses from excitatory to inhibitory neurons on the activity of the (a)

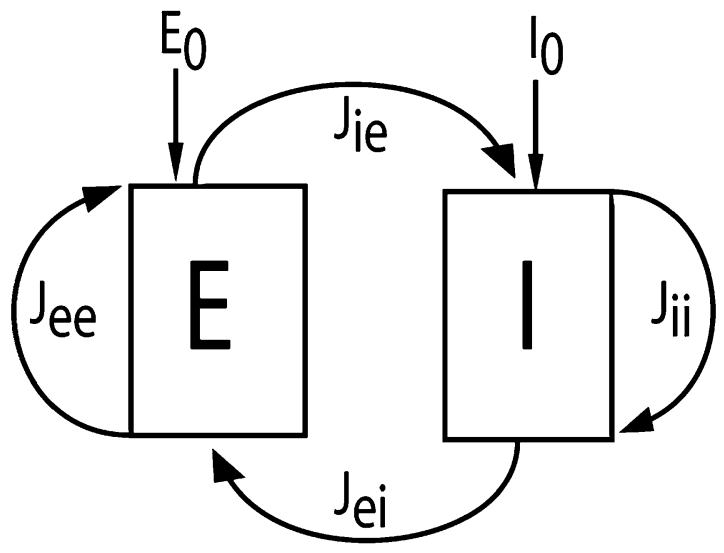

(b)

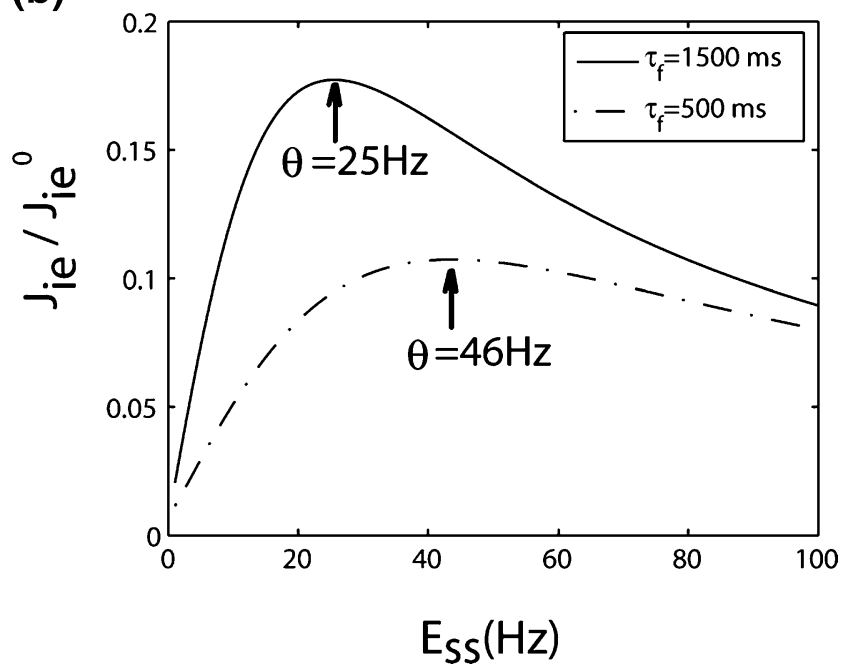

Fig. 1 (a): Schematic representation of a firing rate model of a recurrent network receiving external inputs. $E$ and $I$ represent the mean firing rate of excitatory and inhibitory neuronal subpopulations, respectively. $E_{0}$ and $I_{0}$ represent external input to these subpopulations. $J_{\alpha \beta}$ denotes the average synaptic strength from $\beta$ to $\alpha$. We consider the connection between the excitatory to inhibitory populations, $J_{\text {ie }}$, to be facilitating, whereas the other connections are constants. (b): Examples of two typical curves of the facilitated synaptic strength in a steady state. For a progressive increase in presynaptic firing rates, the effective synaptic strength increases and then decreases owing to simultaneous facilitation of the synaptic utilization fraction, $u$, and a depletion of the synaptic resources, $x$. The result of the facilitating and depressing processes is a bell shaped curve with a peak frequency, $\theta \approx\left(U_{\mathrm{se}} \tau_{\mathrm{r}} \tau_{\mathrm{f}}\right)^{-1 / 2}$ (Markram et al. 1998)

network, we used an activity-dependent model for facilitating synapses described by the following equations (Tsodyks et al. 1998):

$J_{\text {ie }}=J_{\text {ie }}^{0} u x$

$\dot{u}=-\frac{u-U}{\tau_{\mathrm{f}}}+U E(1-u)$

$\dot{x}=\frac{1-x}{\tau_{\mathrm{r}}}-x u E$

$J_{\text {ie }}$ is now an instantaneous effective strength of the facilitating synapse, $x$ and $u$ are variables representing the 
fraction of available synaptic resources and the synaptic utilization fraction, respectively. $J_{\mathrm{ie}}^{0}, U, \tau_{\mathrm{r}}$, and $\tau_{f}$ represent the average absolute efficacy of the synapses, the initial utilization fraction of the synapse, the recovery time constant from the depression, and the facilitating time constant, respectively. This connection could represent the synapse from pyramidal cells to inhibitory interneurons in a cortical neural network. Typical values of such facilitating synapse parameters are $U=0.01, \tau_{f}=1.5 \mathrm{~s}$, and $\tau_{\mathrm{r}}=0.1 \mathrm{~s}$. The parameters representing short-term facilitation are in line with in vitro measurements of pyramidal-interneuron synapses on the rat somatosensory cortex (Thomson et al. 1993; Markram et al. 1998; Wang et al. 1999; Silberberg and Markram 2007). Figure 1(b) illustrates two examples of activity-dependent connections in a steady state calculated from Eq. (3) (Markram et al. 1998). When the firing rate of the presynaptic activity increases, the amplitude of the postsynaptic response is facilitated and then decreases because of dominating depression, reaching a maximal value at the peak frequency, $\theta$. This behavior is the result of the simultaneous facilitation of the synaptic utilization fraction, $u$, and the depression of the synaptic resources, $x$. The value of the peak frequency was approximated for the steady state (Markram et al. 1998):

$\theta=\frac{1}{\sqrt{U \tau_{\mathrm{f}} \tau_{\mathrm{r}}}}$

Figure 1(b) shows the manner in which the parameters determine both the range in which the facilitation process is dominant $\left(E_{\mathrm{ss}}<\theta\right)$, and the sensitivity of the synaptic strength to presynaptic activity (the slope of the facilitating process). Smaller value of any of the parameters, $U, \tau_{f}$ and $\tau_{\mathrm{r}}$ increases the range of facilitation ( $\theta$ is larger) and decrease the slope $\frac{\mathrm{d} J_{\text {ie }}}{\mathrm{d} E_{\mathrm{ss}}}$.

The other synapses in the network were assumed to be static (constant amplitude) since we were interested in elucidating the role of excitatory facilitating connections onto inhibitory interneurons in shaping oscillatory activity.

\section{Facilitation onto interneurons causes sustained oscillations}

The recurrent network described by Eqs. (1), (2) and (3) was analyzed for the different values of the synaptic scaling factor $J_{i e}^{0}$. The rest of the parameters were held constant with values given in Table 1. Figure 2(a) shows a bifurcation diagram for this parameter, revealing four different regimes. For small values of $J_{i e}^{0}$ (I, II) the inhibition cannot regulate the excitation, resulting in runaway firing rate. For a range of intermediate $J_{i e}^{0}<J_{\mathrm{R}}$ ) the network exhibits slow oscillations. For high $J_{i e}^{0}$ values (IV) the network settles into a steady state.
Figure 2(c) presents a trajectory in $\left(E-J_{\mathrm{ie}}\right)$ space, for arbitrarily chosen $J_{\text {ie }}^{0}\left(J_{\mathrm{ie}}^{0}=40 \mathrm{mV} / \mathrm{s}\right)$ between $J_{\mathrm{L}}$ and $J_{\mathrm{R}}$, revealing a limit cycle. The cycle illustrates the periodic alternation between dominancy of excitation and inhibition. The state of the system, represented by $E$ and $J_{i e}$, is rotated in a counterclockwise direction (arrows) around the fixed point (dot). Starting from the point in which $J_{i e}$ is at the minimum (point $\alpha$ ), excitatory feedback increases the firing rate $E$ until the inhibitory feedback $J_{\text {ie }}$ is strong enough to stop this increase $(\beta)$. At this point, the firing rate decreases, but $J_{\text {ie }}$ still increases due to the long time constant of facilitation. Once the excitatory drive to $J_{\text {ie }}$ is low for a sufficiently long time, $J_{\text {ie }}$ starts decreasing $(\chi)$, eventually allowing the firing rate to increase once more due to positive feedback $(\delta)$.

To estimate the $J_{\text {ie }}$ values for which $E$ increases or decreases, note that $E$ has a much faster dynamics than $J_{\text {ie }}$. Therefore $E$ effectively sees a frozen $J_{\text {ie }}$ at each time point, allowing us to estimate the critical $J_{\text {ie }}$ value from such frozen dynamics. We derive this value $\left(J_{\text {ie }}^{\text {th }}\right)$ in Appendix A and plot it as a dotted line in Fig. 2(c), showing that indeed it provides an estimate of the border between divergent and convergent $E$ behavior.

\section{The boundaries of sustained oscillations}

The oscillation region is bound by the region in which excitation diverges, when $J_{\mathrm{ie}}^{0}<J_{\mathrm{L}}$, and by the region in which the excitation converges to a stable fixed point, when $J_{\text {ie }}^{0}>J_{\mathrm{R}}$ (see Fig. 2(a)). We followed these boundary values as a function of different parameters as described in Appendix B. The $\left(J_{\mathrm{ie}}^{0}-\tau_{f}\right)$ parametric space indicates that the oscillation phase exists for a large range of plausible facilitating time constants for the chosen inputs $E_{0}, I_{0}$ and synaptic parameters $U$ and $\tau_{\mathrm{r}}$ (Fig. 3(a)). Both borders show an inverse relationship between $J_{\mathrm{ie}}^{0}$ and $\tau_{f}$. This is because $\tau_{f}$ determines the integration time window of the facilitation,

Table 1 Values of parameters

\begin{tabular}{ll}
\hline Parameters & Value \\
\hline$\tau_{\mathrm{e}}$ & $0.01 \mathrm{~s}$ \\
$\tau_{\mathrm{i}}$ & $0.01 \mathrm{~s}$ \\
$\tau_{\mathrm{r}}$ & $0.1 \mathrm{~s}$ \\
$\tau_{f}$ & $1.5 \mathrm{~s}$ \\
$U$ & 0.01 \\
$E_{0}$ & $19.0 \mathrm{mV}$ \\
$I_{0}$ & $18.1 \mathrm{mV}$ \\
$J_{\text {ee }}, J_{\mathrm{ii}}, J_{\mathrm{ei}}$ & $5,5,9 \mathrm{mV} / \mathrm{Hz}$ \\
$B$ & $0.5 \mathrm{~Hz} / \mathrm{mV}$ \\
$T$ & $15 \mathrm{mV}$ \\
\hline
\end{tabular}


Fig. 2 (a) Bifurcation diagram showing possible steady state values (solid - stable, dashed - unstable) of $E$ as a function of $J_{\mathrm{ie}}^{0}$. The four regimes denoted by roman numerals are: (I) no steady state exists; (II) two unstable steady states; (III) the lower unstable branch $(E<\theta)$ is surrounded by a stable limit cycle (dots mark the minimal and maximal values attained by $E$ in the limit cycle for each value of $J_{\mathrm{ie}}^{0}$ ); (IV) the lower branch is stable. $\theta(25 \mathrm{~Hz})$ is the presynaptic firing rate eliciting the maximal effective strength for $J_{\text {ie }}$ (see Fig. 1(b)). $J_{\mathrm{L}}$ $(27 \mathrm{mV} / \mathrm{s})$ and $J_{\mathrm{R}}(65 \mathrm{mV} / \mathrm{s})$ mark the borders of region III. The diagram was computed using XPPAUT. (b) sample trajectory of slow oscillations. $J_{\mathrm{ie}}^{0}=40 \mathrm{mV} / \mathrm{s}$ giving an oscillation frequency of $1.25 \mathrm{~Hz}$, active state duration $140 \mathrm{~ms}$ and firing rate $18.4 \mathrm{~Hz}$. (c) The trajectory in (b) plotted in $\mathrm{E}-\mathrm{J}_{\text {ie }}$ space showing a limit cycle. The orbit is located around the unstable fixed point (dot). $J_{\mathrm{ie}}^{\text {th }}$ (dotted line) marks the border between the convergent and the divergent regimes as obtained for the "frozen" $\mathrm{J}_{\mathrm{ie}}$ dynamics (see Appendix A). The dynamics proceed counterclockwise as pointed by the arrows. $\alpha, \beta, \chi$ and $\delta$ mark turning points of the cycle as elaborated in the text

and thus a longer time constant results in a larger effective synaptic strength (see Fig. 1(b)).

$J_{\mathrm{L}}$ and $J_{\mathrm{R}}$ borders in the $E_{0}-J_{\text {ie }}^{0}$ parametric space for the value of $\tau_{f}$ are shown in Fig. 3(b). The oscillation phase shrinks rapidly with the increase in $E_{0}$. A horizontal boundary (the dashed line in Fig. 3(b) reflects the minimal amount of external excitatory input which is required to enable the dynamics to overcome the applied constant external input to the inhibitory subpopulation. This line can be precisely calculated by setting $E=0$ in Eq. (1):

$E_{0, \min }=J_{\mathrm{ei}} \frac{I_{0}-T}{1 / \beta+J_{\mathrm{ii}}}+T$

\section{Variety of oscillation forms}

The oscillations can be characterized by the maximal firing rates during the active state, the oscillation frequency, and the amplitude of the silent and active states. The forms of oscillations were examined by performing a series of simulations for various values of $J_{\mathrm{ie}}^{0}$, ranging from the divergent to the convergent borders. The activity near $J_{\mathrm{L}}$, displays the slowest oscillations with long-lasting silent states and wide active state periods with high amplitude (Fig. 3(c1)). The oscillation frequency progressively increases, whereas the amplitude of the active states decreases (Fig. 3(c2-c4)) as $J_{\text {ie }}^{0}$ approaches $J_{\mathrm{R}}$ (Fig. 3(c5)).

We examined the oscillation properties for various parameters of the facilitating synapse $\tau_{f}$ and $J_{\mathrm{ie}}^{0}$, as well as various external inputs, $E_{0}$. Simulations with increasing $\tau_{f}$, $J_{\text {ie }}^{0}$, and $E_{0}$ show a decrease in the maximal firing rate (Fig. 4(a), (d), and (g)). The effect of the first two is not surprising, as they both increase the effectiveness of inhibition. Increasing $E_{0}$ counter intuitively decreases the maximum firing rate of the excitatory population, which is due to the supra linear relationship between the synaptic
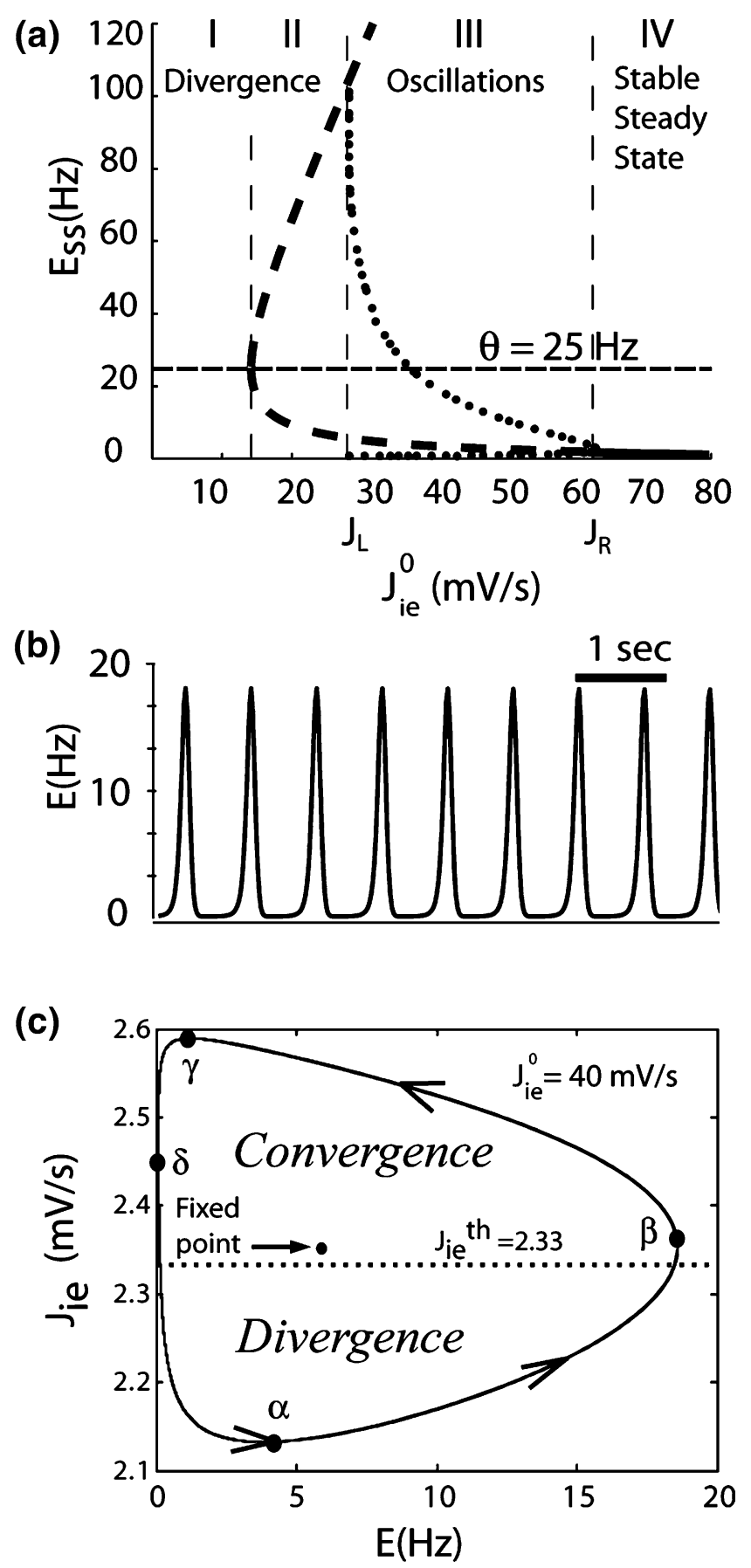

strength of the facilitating synapse and the excitatory firing rate.

The average duration of the active state was quantified by the Full Width Half Maximum (FWHM) of the firing rate in a single cycle. Figure 4(c) shows that the duration of the active state progressively increases with the increase of the synaptic parameters, $U, \tau_{f}$ and $\tau_{\mathrm{r}}$. It spans from 50 to $500 \mathrm{~ms}$ upon varying these parameters. Variations of the $J_{\mathrm{ie}}^{0}$ and $E_{0}$ cause the up state to last between $100 \mathrm{~ms}$ and $300 \mathrm{~ms}$. Generally, an increase of the above-mentioned 

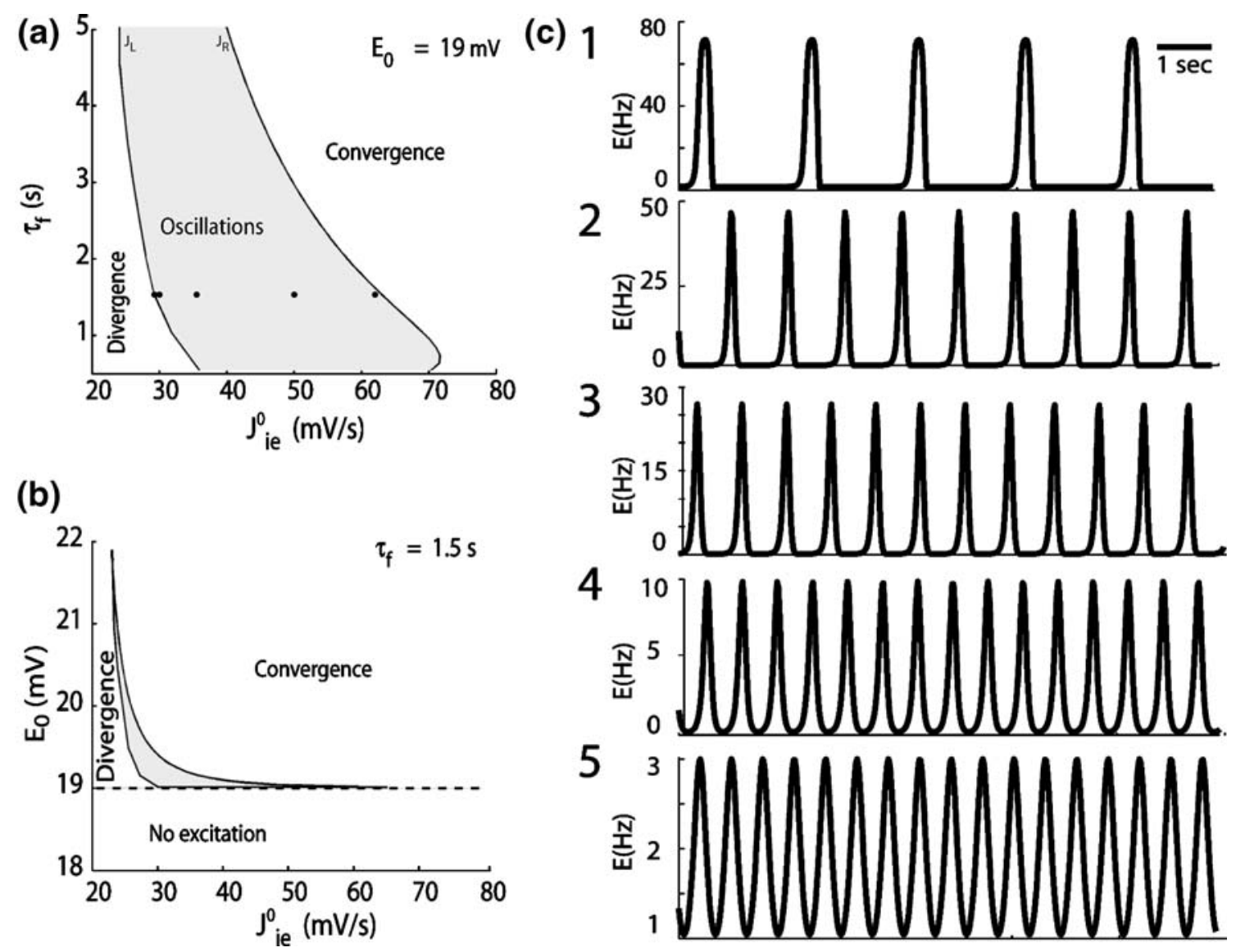

Fig. 3 (a), (b) Parameter space diagrams showing different regimes as in Fig. 2(a). (a) The $\tau_{f}-J_{\text {ie }}^{0}$ parameter space with $E_{0}=19 \mathrm{mV}$ exhibits a wide range in which the network oscillates (gray area). (b) The $E_{0}-J_{\mathrm{ie}}^{0}$ parameter space with $\tau_{f}=1.5 \mathrm{~s}$ exhibits a reduced range of oscillations (gray area). The horizontal dashed line represents the minimal excitatory input required to overcome the inhibition. The value of the minimal input is a solution to the steady-state of Eq. (1) when $E=$

0 . Note that oscillations appear as the first supra threshold solution, and only vanish as excitation is increased. (C1-C5) The excitatory firing rate profiles for progressively increasing absolute synaptic strength, $J_{\mathrm{ie}}^{0}$, for $\tau_{f}=1.5 \mathrm{~s}$ and the external input, $E_{0}=19 \mathrm{mV}$. The oscillation frequency increases while the maximum firing rate decreases. Corresponding black dots appear in panel (a). Note that the scale is different for each figure; see also Fig. 4(d)

synaptic parameters results in a longer $u p$ state duration; however, the up state duration decreases for small $J_{\mathrm{ie}}^{0}$, close to the $J_{\mathrm{L}}$ border (Fig. 4(f)) and the duration also has a minimum when $E_{0}=19.1 \mathrm{mV}$ (Fig. 4(i)). The values of $\tau_{f}$ that give rise to oscillations span over a large range of plausible values (between $1.5 \mathrm{~s}$ and $5 \mathrm{~s}$ ).

\section{Discussion}

Slow oscillations have been revealed in EEG measurements for reduced alertness brain states (Moruzzi and Magoun 1995; Abbott and Blum 1996). They were also observed experimentally in acute cortical slices (Sanchez-Vives and McCormick 2000). We propose a novel mechanism underlying the generation of slow oscillations in the neocortex. A network of excitatory and inhibitory cells with recurrent excitation and facilitating excitatory synapses to the inhibitory population can exhibit slow oscilla- tions. The recurrent excitation initiates the active state by positive feedback. The active state is terminated by inhibition, which is elevated by facilitating excitatory synapses. Simulations and analytical analysis reveal that the properties of these facilitating synapses onto interneurons determine the frequency, duration, and shape of oscillations. Network oscillations depend on the dynamics of facilitating synapses as well as on their absolute strength. Shorter facilitation time constants and a strong connections cause high firing rates and longer periods of oscillation cycles.

7.1 Comparison with previous experimental and theoretical works

In contrast with the model proposed by Timofeev et al. (Timofeev et al. 2000), the initiation mechanism does not depend on spontaneous EPSPs, but rather on recurrent excitation with reduced inhibition, as proposed by Compte 

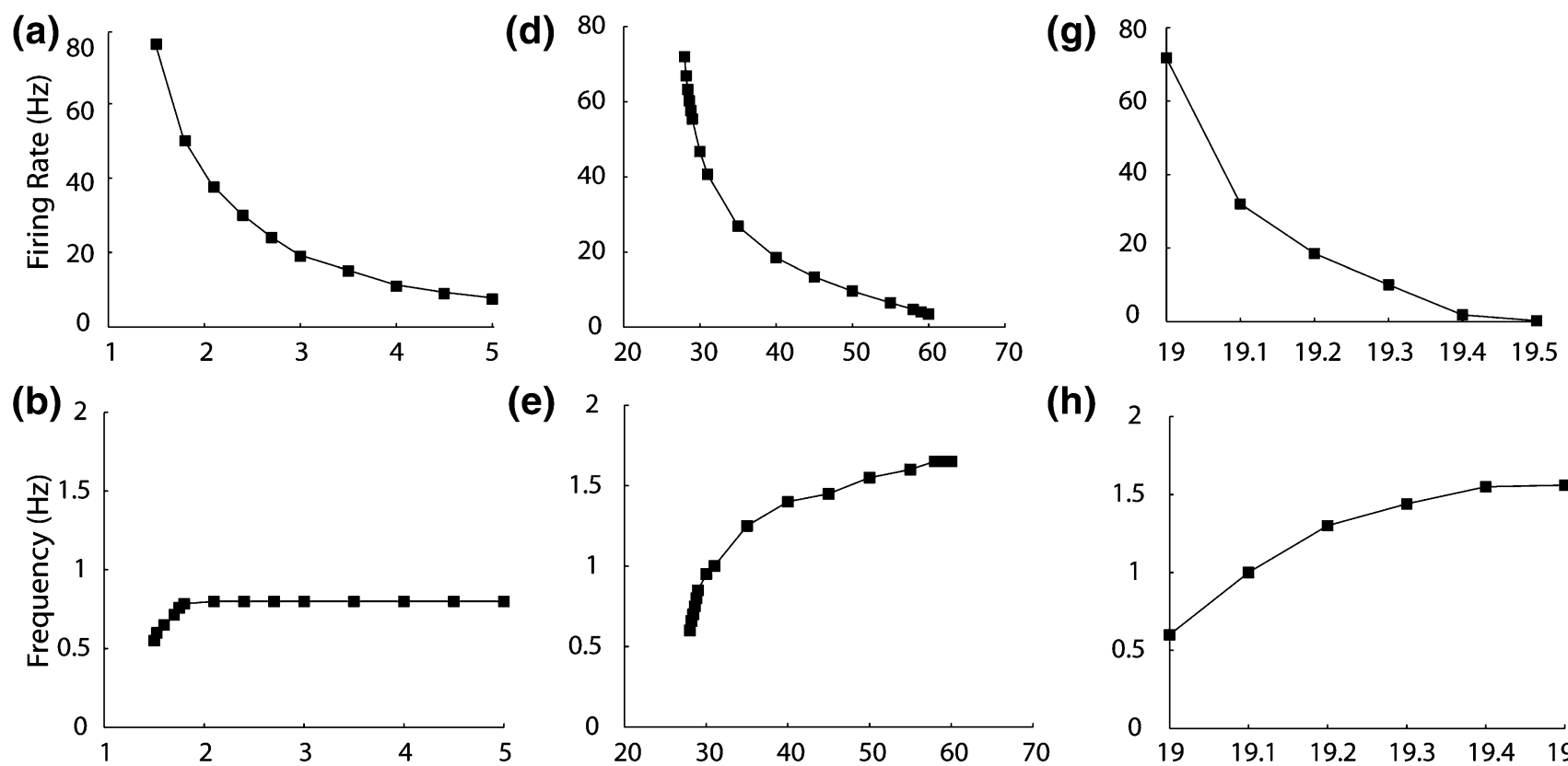

(h)

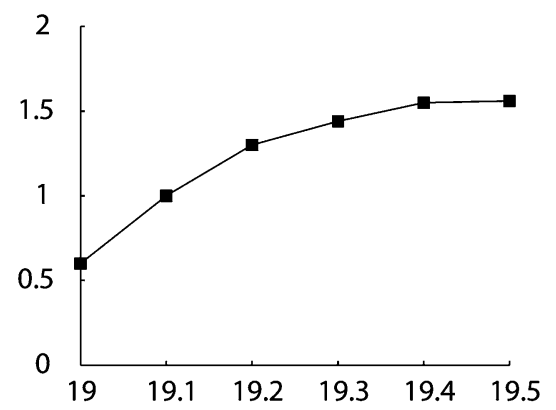

(c)

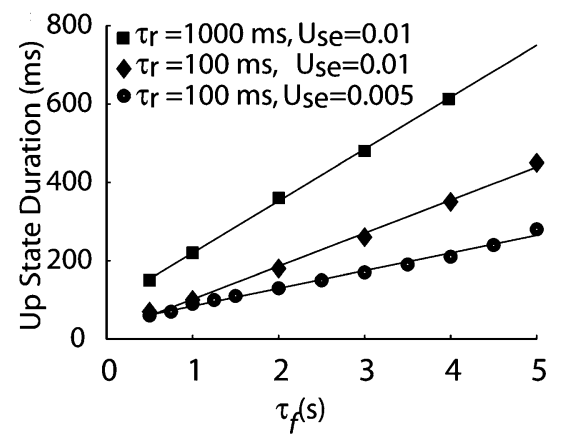

(f)

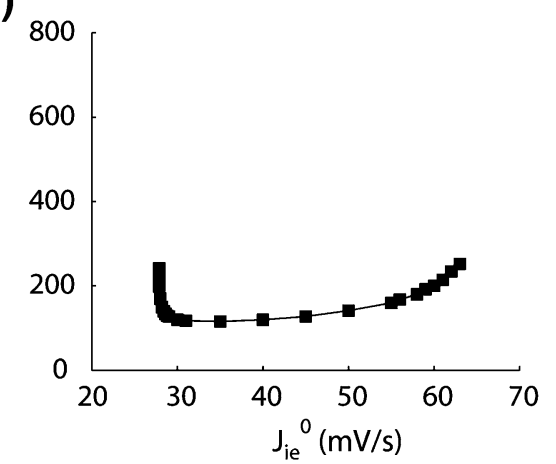

(i)

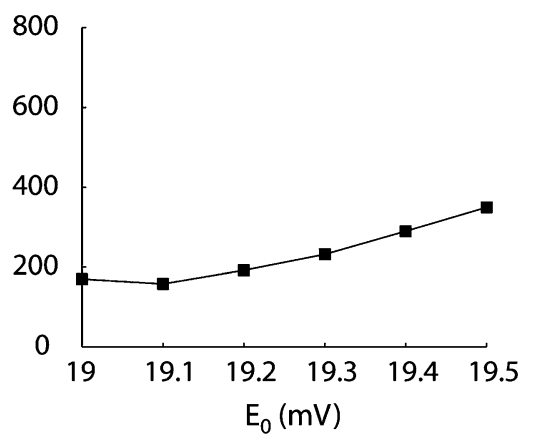

Fig. 4 Characterization of the oscillations when the facilitating synaptic parameters and the external input are varied. (a)-(c) The firing rate (i.e. oscillation amplitude) of the excitatory subpopulations, the oscillation frequency and active state duration (represented by the Full Width Half Maximum-FWHM) is depicted for an increasing facilitation time constant. In panel (c), the facilitating time constant

et al (Compte et al. 2003). The termination mechanism of the active state, occurs in our model due to increased feedback inhibition (as in (Parga and Abbott 2007)), and not by intrinsic neuronal mechanisms, as suggested by the two other models (Timofeev et al. 2000; Compte et al. 2003).

Fig. 5 Dynamic of excitatory and inhibitory input to the excitatory population. The values plotted are the arguments to the $\mathrm{g}$ function in Eq. (1). (a) The ratio between excitatory and inhibitory input is almost constant. The dashed line shows a ratio of 1 . (b) The rise time of both excitation and inhibition is slightly slower than their fall time. One cycle of the active state is shown (a)

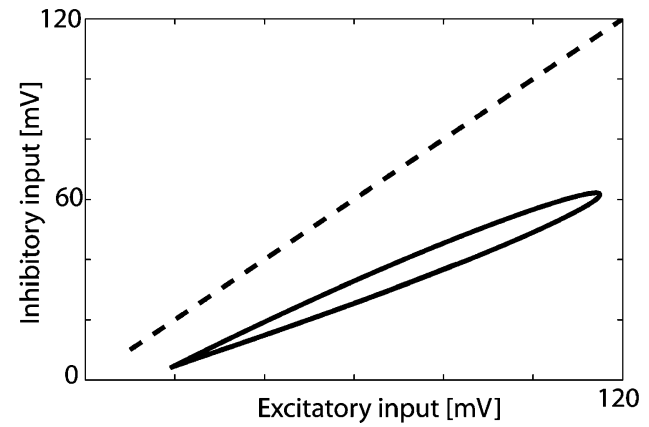

(b)

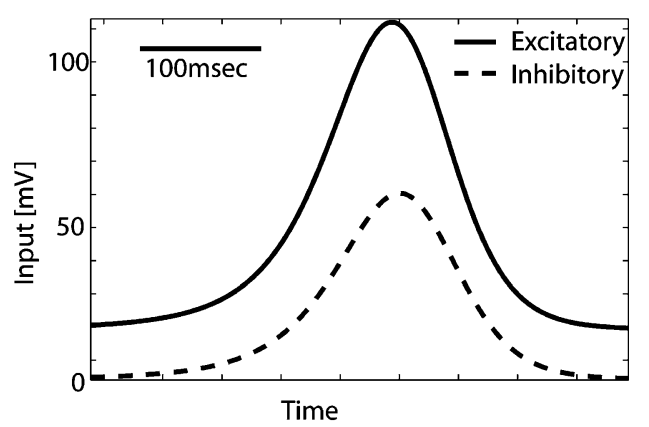


fast increase of the input conductance at the beginning of the active state, followed by a slow decline. Furthermore, the ratio between excitatory and inhibitory conductances remains approximately constant throughout this period (Contreras et al. 1996; Shu et al. 2003). This is in contrast to our description of a gradual increase in inhibition throughout the active state. Figure 5(a) shows that the excitatory/inhibitory ratio in our model is, in fact, almost constant throughout the active state and very similar to that displayed in Shu et al (Fig. 5 there). This stems from the recurrent connections' ability to amplify small differences into large network effects. Regarding the temporal profile, Fig. 5(b) shows that in our case the profile is much more symmetric, with a tendency for a faster decline of conductance. This indeed is opposite to the trend displayed in the above mentioned papers, and is a fundamental property of an oscillation mechanism which is based on facilitation. Possible mechanisms for oscillations can be grossly divided into two categories: declining excitation and elevating inhibition. The former is characterized by a fast increase of conductance which is stopped by depression and slowly recovers. When the increase in excitation stops in the latter mechanism, however, it is due to having been overcome by facilitated inhibition which will quench the remaining excitation very quickly. We therefore propose that conductance measurements in the active state of slow oscillations in different preparations can distinguish the mechanism driving these oscillations.

\subsection{Facilitating excitation of cortical interneurons}

Synaptic connections between pyramidal cells and interneurons are characterized by a wide range of synaptic dynamics (Thomson and Deuchars 1997; Reyes et al. 1998; Wang 1999). Most interneurons receive depressing synapses, yet interneurons that receive facilitating excitatory synapses can be driven to discharge action potentials following high-frequency stimulation, even from a single presynaptic neuron (Markram et al. 1998; Kozloski et al. 2001; Silberberg and Markram 2007). The differences between interneurons receiving depressing excitation and those that receive facilitating excitation are reflected in the conditions under which they will be recruited, as well as the time relative to the excitatory population (Silberberg et al. 2004). Our study suggests a mechanism of recruiting inhibitory neurons via facilitating synapses, resulting in generation of slow oscillations or up and down states.

\subsubsection{Fine tuning of external input}

Figure 3(b) shows that only a small range of external input $\left(\mathrm{E}_{0}\right)$ values can produce oscillations. Note, however, that this range begins at the onset of population activity. As the external input is increased, activity begins in an oscillatory form, and only settles to a steady state as the input is further increased. Possible mechanisms to attain this small suprathreshold input are a balance between excitation and inhibition (Galarreta and Hestrin 1998), or homeostatic plasticity which raises excitation until activity commences (Turrigiano 2007). Another factor which can expand the oscillatory regime is decreasing the synaptic efficacy parameter $U$. As $U$ is decreased, larger values of $E_{0}$ can still sustain slow oscillations. Another concern regarding this fine tuning is whether the phenomenon will still be displayed in a spiking neuron model. Here again, the location of the oscillatory region can provide a straightforward algorithm for finding the relevant parameters simply increasing the excitatory input until activity begins. Such a model is beyond the scope of the current paper.

\subsubsection{Up and down states}

Depolarized-active and hyperpolarized-silent phases recorded in vivo are known as up and down states, respectively. $U p$ and down states have been previously characterized for the neocortex in various studies (Steriade et al. 1993b, 2001; Stern et al. 1997; Lampl et al. 1999; Anderson et al. 2000). Previous studies have suggested that the up state is terminated due to cellular mechanisms such as spike adaptation, inactivation of sodium channels, or flow of hyperpolarizing currents (Sanchez-Vives et al. 2000; Timofeev et al. 2000; Bazhenov et al. 2002). This implies that the properties of oscillations are not determined by the network, but rather by intrinsic neuronal properties. Holcman and Tsodyks (2006) suggested that the transitions between $u p$ and down states are caused by synaptic noise (Holcman and Tsodyks 2006).

Since this is a rate model, we cannot show actual bimodal membrane potential distributions (as in (Parga and Abbott 2007)), and therefore consider a high firing rate of the excitatory population as the $u p$ state and a low firing rate as the down state. Our study suggests that the termination of the $u p$ state is a result of facilitated inhibition and therefore the properties of these slow oscillations depend on the connectivity between the neurons. The common feature of the $u p$ and down states is the existence of distinct phases with high and low discharge frequencies. Facilitating excitatory synapses on inhibitory neurons may therefore play an important role in the regulation of highfrequency events, such as those observed in up and down states or slow oscillations. During high firing rates, facilitating synapses are engaged and the postsynaptic inhibitory population will be activated depending on the presynaptic rate and the synaptic properties. The facilitating synapses do not convey the onset of activity, but become increasingly dominant as activity continues, thus serving as 
a negative feedback mechanism to reduce sustained activity.

\section{Modifications of oscillation forms}

Synchronized oscillations of neural discharges could play a key role in cortical functions such as input selectivity and plasticity, binding cell assemblies and consolidation of learned information (Engel et al. 2001; Buzsaki and Draguhn 2004). These functions require broad diversity of oscillation forms. The present study suggests a qualitative mechanism underlying oscillations that can be tuned by the connectivity parameters of the network. The mean firing rate and the period of oscillation cycle are sensitive to modifications in the absolute synaptic efficacy and to modifications in the time constant of the synaptic facilitation. It has been shown that both of these parameters exhibit large ranges of values (Markram and Tsodyks 1996; Wang et al. 1999). The dynamic parameters of synapses differ across brain areas (Wang et al. 2006), and hence provide an opportunity to measure oscillations in different brain slices and look for correlations between the synaptic parameters and the oscillation properties.

\section{Summary}

We showed that $u p$ and down states can emerge from the interplay between the excitatory and inhibitory populations with excitatory facilitating connection onto inhibitory population. The excitatory neurons elevate the firing rate of the network, which is then suppressed by increasing inhibition recruited by facilitating excitatory connections. Our study predicts that alterations of the facilitating synapses between pyramidal cells and interneurons will affect the oscillations observed in the neocortical microcircuit, as well as the transitions between up and down states. We also point at a fundamental difference between facilitation and depression based mechanisms of oscillation. These predictions can be tested experimentally.

Acknowledgment We thank the anonymous reviewers for their helpful comments. M.T. is partially supported by Israeli Science Foundation, Irving B. Harris Foundation and Abe \& Kathryn Selsky Foundation. G.S. is supported by an HFSP long-term fellowship. O.B. is partially supported by the Azrieli foundation and the Kahn center for system biology.

\section{Appendix A-Stability of dynamics with a "frozen" $J_{i e}$}

When short time scales are considered, the stability of Eq. (1) can be determined as a function of the parameter $J_{i e}$.
We use Eq. (1) in a matrix form for the supra-threshold linear regime.

$$
\begin{aligned}
& \left(\begin{array}{c}
\dot{E} \\
\dot{I}
\end{array}\right)=A\left(\begin{array}{c}
E \\
I
\end{array}\right)+\left(\begin{array}{l}
\frac{\beta}{\tau_{e}}\left(E_{0}-T\right) \\
\frac{\beta}{\tau_{i}}\left(I_{0}-T\right)
\end{array}\right) \\
& A=\left(\begin{array}{ll}
\frac{\beta J_{e e}-1}{\tau_{e}} & \frac{-\beta J_{e i}}{\tau_{e}} \\
\frac{\beta J_{i e}}{\tau_{i}} & \frac{-\beta J_{i i}-1}{\tau_{i}}
\end{array}\right)
\end{aligned}
$$

The condition for stability is a negative trace and positive determinant. Since Trace(A) is independent of $J_{i e}$, we chose the simulation parameters (see Table 1) which render it negative, thus allowing stability for some $J_{i e}$ values. The stability is thus determined by $\operatorname{Det}(\mathrm{A})$, defining $J_{i e}^{\text {th }}$ as a border between the stable and the unstable regimes:

$J_{i e}^{t h}=\frac{\left(\beta J_{e e}-1\right)\left(\beta J_{i i}+1\right)}{\beta^{2} J_{e i}}$

$J_{i e}^{\text {th }}$ represents the criterion for stability as follows: when $J_{i e}>J_{i e}^{t h}, \mathrm{E}$ and I relax to a constant, and when $J_{i e}>J_{i e}^{\text {th }}$, the solution diverges.

\section{Appendix B-estimating the oscillation borders}

The right border of the oscillation regime $\left(J_{\mathrm{R}}\right.$ in Fig. 2(a)) can be calculated by searching for the $J_{i e}^{0}$ value for which the steady state becomes stable. This can be done by calculating the eigenvalues of the linearized dynamics at the steady state:

$$
\left(\begin{array}{cccc}
\frac{\beta J_{e e}-1}{\tau_{e}} & \frac{-\beta J_{e i}}{\tau_{e}} & 0 & 0 \\
\frac{\beta J_{i e}^{0} x_{s s} u_{s s}}{\tau_{i}} & \frac{-\left(\beta J_{i i}+1\right)}{\tau_{i}} & \frac{\beta J_{i e}^{0} x_{s s} E_{s s}}{\tau_{i}} & \frac{\beta J_{i e}^{0} u_{s s} E_{s s}}{\tau_{i}} \\
U\left(1-u_{s S}\right) & 0 & -\left(\frac{1}{\tau_{f}}+E_{s s} U\right) & 0 \\
-x_{s s} u_{s s} & 0 & -x_{S S} E_{s S} & -\left(\frac{1}{\tau_{r}}+E_{s s} u_{s s}\right)
\end{array}\right)
$$

where $x_{s s}, u_{s s}, E_{s s}$ are the steady state values.

The left border of the oscillation regime ( $\mathrm{J}_{\mathrm{L}}$ in Fig. 2(a)) is defined by a divergence of the oscillations, and was thus searched for by simulating the network until the border between a stable limit cycle and divergent behavior was found.

\section{References}

Abbott, L. F., \& Blum, K. I. (1996). Functional significance of longterm potentiation for sequence learning and prediction. Cerebral Cortex, 6, 406-416.

Anderson, J., Lampl, I., Reichova, I., Carandini, M., \& Ferster, D. (2000). Stimulus dependence of two-state fluctuations of membrane potential in cat visual cortex. Nature Neuroscience, 3, 617621. 
Bazhenov, M., Timofeev, I., Steriade, M., \& Sejnowski, T. J. (2002). Model of thalamocortical slow-wave sleep oscillations and transitions to activated States. Journal of Neuroscience, 22, 8691-8704.

Buzsaki, G., \& Draguhn, A. (2004). Neuronal oscillations in cortical networks. Science, 304, 1926-1929.

Compte, A., Sanchez-Vives, M. V., McCormick, D. A., \& Wang, X. J. (2003). Cellular and network mechanisms of slow oscillatory activity $(<1 \mathrm{~Hz})$ and wave propagations in a cortical network model. Journal of Neurophysiology, 89, 2707-2725.

Contreras, D., \& Steriade, M. (1995). Cellular basis of EEG slow rhythms: a study of dynamic corticothalamic relationships. Journal of Neuroscience, 15, 604-622.

Contreras, D., Timofeev, I., \& Steriade, M. (1996). Mechanisms of long-lasting hyperpolarizations underlying slow sleep oscillations in cat corticothalamic networks. Journal of Physiology, 494( $\mathrm{Pt}$ 1), 251-264.

Engel, A. K., Fries, P., \& Singer, W. (2001). Dynamic predictions: oscillations and synchrony in top-down processing. Nature Reviews Neuroscience, 2, 704-716.

Galarreta, M., \& Hestrin, S. (1998). Frequency-dependent synaptic depression and the balance of excitation and inhibition in the neocortex. Nature Neuroscience, Plos, 1, 587-594.

Holcman, D., \& Tsodyks, M. (2006). The emergence of up and down states in cortical networks. Plos. 2, e23.

Kozloski, J., Hamzei-Sichani, F., \& Yuste, R. (2001). Stereotyped position of local synaptic targets in neocortex. Science, 293, 868 872.

Lampl, I., Reichova, I., \& Ferster, D. (1999). Synchronous membrane potential fluctuations in neurons of the cat visual cortex. Neuron, $22,361-374$

Markram, H., \& Tsodyks, M. (1996). Redistribution of synaptic efficacy between neocortical pyramidal neurons. Nature, 382, 807-810.

Markram, H., Wang, Y., \& Tsodyks, M. (1998). Differential signaling via the same axon of neocortical pyramidal neurons. Proceedings of the National Academy of Sciences of the United States of America, 95, 5323-5328.

Moruzzi, G., \& Magoun, H. W. (1995). Brain stem reticular formation and activation of the EEG. 1949. Journal of Neuropsychiatry and Clinical Neurosciences, 7, 251-267.

Parga, N., \& Abbott, L. F. (2007). Network model of spontaneous activity exhibiting synchronous transitions between up and down states. Frontiers in Neuroscience, 1, 57-66.

Reyes, A., Lujan, R., Rozov, A., Burnashev, N., Somogyi, P., \& Sakmann, B. (1998). Target-cell-specific facilitation and depression in neocortical circuits. Nature Neuroscience, 1, 279-285.

Sanchez-Vives, M. V., \& McCormick, D. A. (2000). Cellular and network mechanisms of rhythmic recurrent activity in neocortex. Nature Neuroscience, 3, 1027-1034.

Sanchez-Vives, M. V., Nowak, L. G., \& McCormick, D. A. (2000). Cellular mechanisms of long-lasting adaptation in visual cortical neurons in vitro. Journal of Neuroscience, 20, 4286-4299.
Shu, Y., Hasenstaub, A., \& McCormick, D. A. (2003). Turning on and off recurrent balanced cortical activity. Nature, 423, 288-293.

Silberberg, G., \& Markram, H. (2007). Disynaptic inhibition between neocortical pyramidal cells mediated by Martinotti cells. Neuron, $53,735-746$.

Silberberg, G., Wu, C., \& Markram, H. (2004). Synaptic dynamics control the timing of neuronal excitation in the activated neocortical microcircuit. Journal of Physiology, 556, 19-27.

Steriade, M., Amzica, F., \& Contreras, D. (1996). Synchronization of fast $(30-40 \mathrm{~Hz})$ spontaneous cortical rhythms during brain activation. Journal of Neuroscience, 16, 392-417.

Steriade, M., McCormick, D. A., \& Sejnowski, T. J. (1993a). Thalamocortical oscillations in the sleeping and aroused brain. Science, 262, 679-685.

Steriade, M., Nunez, A., \& Amzica, F. (1993b). A novel slow $(<1 \mathrm{~Hz})$ oscillation of neocortical neurons in vivo: Depolarizing and hyperpolarizing components. Journal of Neuroscience, 13, 3252 3265 .

Steriade, M., Timofeev, I., \& Grenier, F. (2001). Natural waking and sleep states: A view from inside neocortical neurons. Journal of Neurophysiology, 85, 1969-1985.

Stern, E. A., Kincaid, A. E., \& Wilson, C. J. (1997). Spontaneous subthreshold membrane potential fluctuations and action potential variability of rat corticostriatal and striatal neurons in vivo. Journal of Neurophysiology, 77, 1697-1715.

Thomson, A. M., \& Deuchars, J. (1997). Synaptic interactions in neocortical local circuits: Dual intracellular recordings in vitro. Cerebral Cortex, 7, 510-522.

Thomson, A. M., Deuchars, J., \& West, D. C. (1993). Single axon excitatory postsynaptic potentials in neocortical interneurons exhibit pronounced paired pulse facilitation. Neuroscience, 54, 347-360.

Timofeev, I., Grenier, F., Bazhenov, M., Sejnowski, T. J., \& Steriade, M. (2000). Origin of slow cortical oscillations in deafferented cortical slabs. Cerebral Cortex, 10, 1185-1199.

Tsodyks, M., Pawelzik, K., \& Markram, H. (1998). Neural networks with dynamic synapses. Neural Computation, 10, 821-835.

Turrigiano, G. (2007). Homeostatic signaling: The positive side of negative feedback. Current Opinion in Neurobiology, 17, 318324.

Wang, X. J. (1999). Synaptic basis of cortical persistent activity: The importance of NMDA receptors to working memory. Journal of Neuroscience, 19, 9587-9603.

Wang, Y., Gupta, A., \& Markram, H. (1999). Anatomical and functional differentiation of glutamatergic synaptic innervation in the neocortex. Journal of Physiology (Paris), 93, 305-317.

Wang, Y., Markram, H., Goodman, P. H., Berger, T. K., Ma, J., \& Goldman-Rakic, P. S. (2006). Heterogeneity in the pyramidal network of the medial prefrontal cortex. Nature Neuroscience, 9, $534-542$.

Wilson, H. R., \& Cowan, J. D. (1972). Excitatory and inhibitory interactions in localized populations of model neurons. Biophysical Journal, 12, 1-24. 